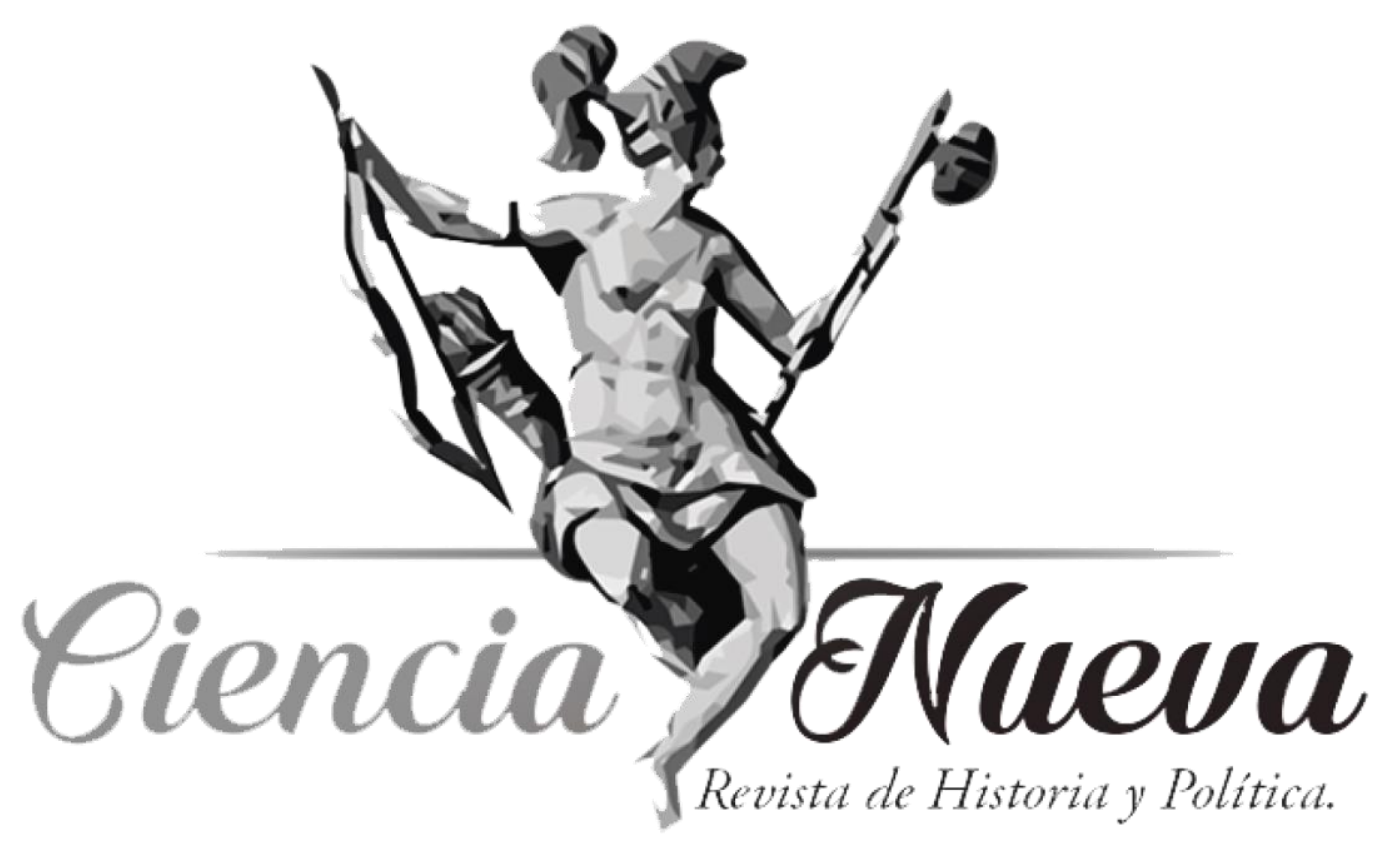

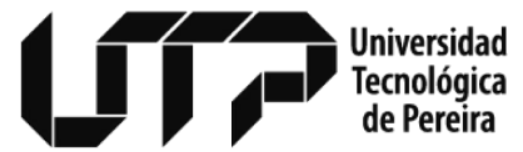

Maestría en Historia

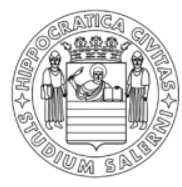

UNIVERSITÀ DEGLI STUDI DI SALERNO

Maestría en Ciencia Política

RESEÑAS

RESEÑA DE TESIS DOCTORAL: CORREA RAMÍREZ, JHON JAIME. CIVISMO Y EDUCACIÓN EN PEREIRA Y MANIZALES (1925-1950): UN ANÁLISIS COMPARATIVO ENTRE SUS SOCIABILIDADES, VISIONES DE CIUDAD Y CULTURA CÍVICA. PEREIRA: EDITORIAL UNIVERSIDAD TECNOLÓGICA

DE PEREIRA, 2015. 270 PP.

REVIEW OF DOCTORAL THESIS: CORREA RAMÍREZ, JHON JAIME. CITIZENSHIP AND EDUCATION IN PEREIRA AND MANIZALES (1925-1950): COMPARATIVE ANALYSIS OF SOCIABILITIES, VISIONS OF CITY AND CIVIC CULTURE. PEREIRA: EDITORIAL OF UNIVERSIDAD TECNOLÓGICA DE PEREIRA, 2015, 270 PP.

Vol. 2 Núm. 2, Julio-Diciembre de 2018

Pereira, Colombia 


\section{RESEÑA DE TESIS DOCTORAL: CORREA RAMÍREZ, JHON JAIME. CIVISMO Y EDUCACIÓN EN PEREIRA Y MANIZALES (1925-1950): UN ANÁLISIS COMPARATIVO ENTRE SUS SOCIABILIDADES, VISIONES DE CIUDADY CULTURA CÍVICA. PEREIRA: EDITORIAL UNIVERSIDAD TECNOLÓGICA DE PEREIRA, 2015. 270 PP.** \\ REVIEW OF DOCTORAL THESIS: CORREA RAMÍREZ, JHON JAIME. CITIZENSHIP AND EDUCATION IN PEREIRA AND MANIZALES (1925-1950): COMPARATIVE ANALYSIS OF SOCIABILITIES, VISIONS OF CITY AND CIVIC CULTURE. PEREIRA: EDITORIAL OF UNIVERSIDAD TECNOLÓGICA DE PEREIRA, 2015, 270 PP.}

Javier Jaramillo Vélez** javijara136@yahoo.com

ORCID: http://orcid.org/0000-0001-6797-4027

\begin{aligned} & \hline Recibido: 25 de febrero de 2018. \\ & Revisado: 13 de agosto de 2018. \\ & Aceptado: 27 de agosto de 2018. \\ & Publicado: 23 de noviembre de 2018. \\ & \hline\end{aligned}

$\mathrm{E}$ 1 presente escrito tiene como propósito fundamental presentar unas breves reflexiones con un carácter descriptivo-analítico sobre algunos apartes de la tesis doctoral del profesor John Jaime Correa Ramírez, Civismo y Educación en Pereira y Manizales (1925-1950): Un análisis comparativo entre sus sociabilidades, visiones de ciudad y cultura cívica. De manera especial se analizó el capítulo II referido al ornato público: la visión estética y civilizada de la ciudad, escrito trabajado en clase durante el Seminario de Historia Regional y Urbana con el doctor Enrique Rodríguez Caporalli. Para alcanzar un mejor nivel de comprensión conceptual se hizo necesario abordar la lectura de las temáticas del primer capítulo, que desarrollan los procesos del cambio en la configuración urbana de las ciudades, un marco teórico referencial para el tema del civismo y la acción de las sociabilidades cívicas.

De igual manera, se recurre a la lectura del artículo del doctor Correa "El discurso del civismo en Pereira o la 'sacralidad' de lo público durante el siglo XX"1. Es un texto esclarecedor para esta reseña, con miras a elucidar algunos elementos conceptuales en el abordaje teórico del problema de investigación que nos ocupa, en la línea de investigación recorrida por Correa Ramírez en los últimos años de su quehacer académico.

El trabajo de investigación del profesor John Jaime Correa, presentado como tesis doctoral, es un estudio comparativo entre los procesos de desarrollo histórico de dos ciudades del llamado Eje Cafetero o Triángulo del Café, Manizales y Pereira para el periodo comprendido entre 1925 y 1950, tomando como criterios de periodización para su

\footnotetext{
${ }^{*}$ El presente artículo respeta las directrices y normas dispuestas en la Declaración de Ética de Publicación de Ciencia Nueva, Revista de Historia y Política. Esta declaración puede consultarse en la página web de la revista: revistas.utp.edu.co/index.php/historia.

${ }^{* *}$ Licenciado en Ciencias Sociales, especialista en Historia Contemporánea de Colombia y Desarrollos Regionales y magíster en Historia por la Universidad Tecnológica de Pereira. Docente de la Institución Educativa Lorencita Villegas de Santos del municipio de Santa Rosa de Cabal, Risaralda.

${ }^{1}$ John Jaime Correa Ramírez, "El discurso del civismo en Pereira o la 'sacralidad' de lo público durante el siglo XX", HiSTORelo 1, n. 2 (2009): 7-31, ISSN: 2145-132X.
} 
delimitación temporal la fundación de la Sociedad de Mejoras Públicas de Pereira en 1925 hasta $1950^{2}$. Temporalidad conocida como la época de oro de ambas instituciones por su auge en la construcción de obras materiales en el desarrollo urbanístico, embellecimiento de las localidades con la construcción de obras públicas de gran impacto social y el diseño de dispositivos de control social por parte de los grupos hegemónicos que detentaban el poder económico y político sobre grandes masas de población urbana y los migrantes que llegaban especialmente de zonas de violencia (Cundinamarca y Boyacá) o de los espacios rurales de municipios aledaños. El autor sintetiza el objetivo de la obra en los siguientes términos:

El principal objetivo de la tesis se centra en comparar la incidencia de las sociabilidades cívicas, los proyectos de modernización, los discursos sobre el progreso y la educación moral y cívica de los ciudadanos, en el desarrollo de la historia urbana de Pereira y Manizales (19251950), época considerada como la de mayor relieve en cuanto al papel que las entidades cívicas de estas ciudades desempeñaron en relación con el desarrollo "espiritual y moral" de sus respectivas urbes ${ }^{3}$.

Para el desarrollo de la investigación se recurre fundamentalmente al uso de fuentes primarias en archivos municipales, las hemerotecas de las dos ciudades y el rastreo de información en los principales diarios de circulación de la época: La Patria de Manizales, creado en 1921 periódico de corte "cívico-partidista" y El Diario de Pereira fundado en 1929. Cada uno respondía a una orientación partidista, conservadora en Manizales y liberal para el caso de Pereira, lo que enardecía aún más las tensiones y controversias entre los dos centros urbanos que se disputaban el predominio como polos de desarrollo en el centro-occidente colombiano.

El rastreo de fuentes permite presentar un informe de investigación muy bien documentado con archivos fotográficos de época, con todo lo que representa para la interpretación histórica este tipo de documentos en la comprensión semántica de los acontecimientos. Al igual que una reseña cartográfica que da cuenta de la evolución del territorio en las primeras décadas del siglo XX, especialmente en el crecimiento del perímetro urbano asociado al incremento significativo de la población debido a la dinámica en el comportamiento de las variables demográficas de la época.

La investigación desde el punto de vista metodológico es un estudio histórico comparado, desarrolla técnicas que van desde la contrastación de documentos de época en diarios y archivos de entidades públicas, hasta la lectura sistemática y crítica de autores que han trabajado la historia local. Por citar algunos: Jaime Jaramillo Uribe, quien público con motivo del centenario de Pereira un texto con Luis Duque Gómez y Juan Friede (1963); Carlos Echeverry Uribe, Fernando Uribe Uribe, Hugo Ángel Jaramillo, Víctor Zuluaga, Rigoberto Gil, Albeiro Valencia, entre otros. Algunos de estos escritores direccionaron sus trabajos hacia el estilo de la crónica.

El proceso de hermenéutica en el tratamiento de los documentos, le permite al profesor Correa Ramírez asumir distancia epistemológica frente a una serie de mitos urbanos que han posicionado a estas ciudades en el rol de adalides del civismo y el progreso, como paradigmas fundantes de una modernidad tardía en nuestro territorio y que se inaugura en la

\footnotetext{
${ }^{2}$ Es pertinente anotar que la Sociedad de Mejoras Públicas de Manizales se fundó en 1912.

3 Jhon Jaime Correa Ramírez, Civismo y educación en Pereira y Manizales (1925-1950): Un análisis comparativo entre sus sociabilidades, visiones de ciudad y cultura cívica (tesis doctoral, Universidad Tecnológica de Pereira, 2014), 11.
} 
Europa decimonónica con postulados positivistas y para Colombia sufren una hibridación con la cosmovisión judeocristiana y escatológica del mundo. A este respecto afirma el autor en su andadura investigativa:

La eficacia del discurso cívico se apoyó en la exaltación de valores morales de profundo arraigo cristiano, que permiten entender el civismo como un coherente sistema cultural de significación, a la manera de una "religión civil", que era necesario promover en la vida pública como privada, a través de sus diferentes códigos de significación y de puesta en escena $^{4}$.

En este orden de ideas el autor muestra la importancia del clero en las gestas cívicas de cada ciudad, aun antes que la escuela, la administración pública con la precariedad de su erario y los escasos medios de difusión cultural como la prensa hablada o escrita. El clero hace irrupción desde el pulpito con un discurso no solo moralizante y de control ideológico, sino con un entramado de ideas que jalonan a través de convites, ferias, bazares y veladas, la ejecución de importantes obras de bienestar y progreso a la comunidad, caso concreto el hospital San Jorge de Pereira, la Villa Olímpica, el Aeropuerto Matecaña:

Desde la liturgia y el púlpito se promovieron las primeras esferas públicas en relación con el desarrollo de las ciudades y el compromiso de una ciudadanía participativa, mucho antes de que aparecieran los primeros medios de opinión pública ${ }^{5}$.

De esta manera las élites van creando diferentes clubes y sociedades de beneficencia con un discurso altruista y filantrópico que moviliza a grandes masas de población: Club Rialto, Club Rotario, Club del Comercio, donde se reúnen las élites a trazar los rumbos de progreso y civilidad de cada ciudad, en muchas ocasiones en una férrea oposición política al excesivo centralismo de los gobiernos de la capital de la república. Aspecto que fue duramente criticado por algunos sectores sociales opuestos al discurso conservatizante de los grupos institucionalizados, porque en determinados momentos de su actividad emprendedora eximieron al Gobierno nacional de su responsabilidad en la construcción de obras públicas en estos territorios locales.

Es fundamental en las tesis expresadas por el profesor Correa, la ruptura que presenta en relación con la visión rosa y movilizadora del civismo en Manizales y Pereira, a causa de las tensiones y los conflictos sociales generados por las medidas draconianas impuestas a los ciudadanos por las juntas de notables y sociedades cívicas con el aval de las autoridades:

Cuando la vida pública se organiza en torno a los valores del civismo, es normal que se impongan unos recios valores morales, que incluso son acompañados de numerosas sanciones civiles para quienes afecten el orden o el ornato público ${ }^{6}$.

En igual sentido, el interés de las élites por imponer estructuras arquitectónicas modernizantes en las obras públicas de cada ciudad e instaurar patrones culturales de control social a través de las llamadas "buenas costumbres", hábitos de higiene social y personal,

\footnotetext{
${ }^{4}$ John Jaime Correa Ramírez, "El discurso del civismo en Pereira o la 'sacralidad' de lo público durante el siglo XX", 11.

${ }^{5}$ Jhon Jaime Correa Ramírez, Civismo y educación en Pereira y Manizales (1925-1950), 13.

${ }^{6}$ Jhon Jaime Correa Ramírez, Civismo y educación en Pereira y Manizales (1925-1950), 20.
} 
"buenos modales" y otros, entraban en franca contradicción con pautas de comportamiento social ancestralmente entronizadas en la cultura campesina. Lo que generaba ambientes hostiles por las frecuentes prohibiciones y destrucciones asociadas a las medidas de todo orden, implementadas con el respaldo de los gobiernos municipales:

[...] en otras ocasiones los llamados cívicos tenían como fin incentivar la destrucción de los platanales ubicados dentro del perímetro urbano y adelantar medidas restrictivas para controlar el paso de las recuas de mulas por las calles céntricas de la ciudad, en una clara muestra de querer eliminar los vestigios semirurales de la faz urbana ${ }^{7}$.

La narrativa implementada es expositiva y presenta en orden cronológico los fenómenos desde un enfoque dialéctico, dilucidando la multicausalidad de los acontecimientos históricos, estableciendo analogías entre los procesos históricos de una y otra ciudad, que es el propósito central del proyecto de investigación. De manera que encuentra conexiones y rupturas entre los diferentes escenarios socioculturales, económicos y políticos, para dar cuenta de una veta de investigación que perfila en perspectiva otros filones de indagación de un periodo muy poco estudiado en la historia urbana de Pereira y Manizales.

El desarrollo argumentativo presenta categorías muy propias de los enfoques transdisciplinarios en el ámbito de las ciencias sociales, acudiendo a la migración de conceptos propios de la historia, la sociología, la antropología, la economía, la filosofía entre otras. Como expresa el historiador español Julio Aróstegui: "El nombre de los fenómenos y las categorías que estudia la historiografía han sido acuñados muy frecuentemente en otras ciencias". Lo que le permite al profesor Correa en su investigación ir tejiendo entramados de significación que dan cuenta de la realidad social estudiada. Recorre unos conceptos centrales y la definición que se da a lo largo del texto: civismo, sociabilidad, educación, sacralidad, modernidad, progreso, civilidad, tradición, cultura, discurso, ciudad, élites, rituales, imaginarios colectivos, inclusión, exclusión, vida pública, entre otros.

Se puede relacionar con el ámbito de la historia urbana en otras fuentes investigativas, en tanto ha sido una constante historica en Colombia y América Latina, la rivalidad ancestral existente entre pueblos y ciudades que se disputan la hegemonía en determinadas áreas de influencia, lo que se traduce en importantes beneficios de orden económico (industrial, comercial) o cultural para sus habitantes, particularmente para las élites que detentan el poder.

En este orden de ideas, el discurso político e ideológico del civismo presenta como recurrencia la alusión a la perpetua crisis del presente, donde se quiere demostrar de manera descarnada una cruda realidad que en ciertos momentos no corresponde a los hechos, con la pretensión de exaltar aún más las sinergias y generar sentimientos de culpa que impulsen con mayor dinamismo el accionar ciudadano. A este respecto el autor afirma:

En muchas ocasiones la predica cívica echaba mano del recurso de la crisis o decaimiento del civismo como una forma de volver a despertar el fervor y el compromiso ciudadano con algunos frentes de trabajo público que desempeñaban las SMP de las dos ciudades ${ }^{8}$.

\footnotetext{
${ }^{7}$ Jhon Jaime Correa Ramírez, Civismo y educación en Pereira y Manizales (1925-1950), 123.

${ }^{8}$ Jhon Jaime Correa Ramírez, Civismo y educación en Pereira y Manizales (1925-1950), 125.
} 
Más adelante el investigador señala a este respecto:

Es tan frecuente encontrar estas alusiones a la crisis del civismo en las diversas fuentes consultadas que en ocasiones se puede llegar a dudar si realmente hubo un momento de auge o esplendor de este proceso cívico o si siempre se mantuvo en crisis ${ }^{9}$.

Para finalizar, es importante destacar, que los temas históricos de indagación y reflexión académica no se agotan con la publicación historiográfica, por el contrario, se convierten en una inagotable fuente de problematización permanente, donde hacen irrupción nuevos actores sociales, nuevas urdimbres, innovadores escenarios de tensión y confrontación social. Este proceso inacabado es lo que construye sentidos y hace más apasionante el oficio del historiador. Por lo anterior la obra del Profesor Correa es pionera en el tema de investigación y abre puertas para futuras problemáticas de investigación social en las líneas de historia regional y urbana.

\section{Conclusiones}

El abordaje teórico de la tesis del doctor Correa Ramírez permite comprender con significativa claridad, de manera amplia y documentada, los intereses de los grupos de poder que se van formando y consolidando entorno a las sociedades cívicas, lo mismo que los mecanismos a los que recurren para legitimarse dentro de una sociedad determinada y conquistar las metas cívicas proyectadas.

La lectura posibilita evidenciar cómo ha sido una constante histórica para confrontar a algunos sectores sociales resistentes al cambio y la modernización, acudir a la muy manida frase "Todo tiempo pasado fue mejor". Una añoranza por parte de los entes gubernamentales o élites sociales que acuden al fervor ciudadano, enalteciendo sentimientos patrios en la población para jalonar políticas públicas en determinadas coyunturas históricas. Por ello, el civismo como discurso institucional y retórica de movilización social, se resiste a desaparecer en vastas zonas del territorio colombiano, buscando institucionalizarse en los currículos de los centros educativos, a través del rescate de la educación cívica y la urbanidad con el desarrollo de las llamadas competencias ciudadanas, política educativa emanada desde el Ministerio de Educación Nacional.

\footnotetext{
${ }^{9}$ Jhon Jaime Correa Ramírez, Civismo y educación en Pereira y Manizales (1925-1950), 125.
} 


\section{Bibliografía}

Correa Ramírez, John Jaime. "El discurso del civismo en Pereira o la 'sacralidad' de lo público durante el siglo XX”. HiSTORelo 1, n. ${ }^{\circ} 2$ (2009): 7-31, ISSN: 2145-132X.

. Civismo y educación en Pereira y Manizales (1925-1950): un

análisis comparativo entre sus sociabilidades, visiones de ciudad y cultura cívica. Pereira: Editorial Universidad Tecnológica de Pereira, 2015. 270 pp. 